Article

\title{
A Microwave-Assisted Bismuth Nitrate-Catalyzed Unique Route Toward 1,4-Dihydropyridines
}

\author{
Debasish Bandyopadhyay, Stephanie Maldonado ${ }^{\dagger}$ and Bimal K. Banik * \\ Department of Chemistry, The University of Texas-Pan American, 1201 West University Drive, \\ Edinburg, TX 78539, USA \\ $\dagger$ Undergraduate research participant. \\ * Author to whom correspondence should be addressed; E-Mail: banik@utpa.edu; \\ Tel.: +1-956-665-8741; Fax: +1-956-384-5006.
}

Received: 6 February 2012; in revised form: 26 February 2012 / Accepted: 27 February 2012 / Published: 5 March 2012

\begin{abstract}
The classical Hantzsch reaction is one of the simplest and most economical methods for the synthesis of biologically important and pharmacologically useful 1,4-dihydropyridine derivatives. Bismuth nitrate pentahydrate under microwave irradiation is proven to act as a very efficient catalyst for a one-pot, three-component synthesis of 1,4-dihydropyridines in excellent yields from diverse amines/ammonium acetate, aldehydes and 1,3-dicarbonyl compounds within 1-3 min under solvent-free conditions. The present environmentally benign procedure for the synthesis of 1,4-dihydropyridines is suitable for library synthesis and it will find application in the synthesis of potent biologically active molecules. The excellent yield and extreme rapidity of the method is due to a concurrent effect of the catalyst and microwave irradiation.
\end{abstract}

Keywords: microwave; 1,4-dihydropyridines; catalysis; green synthesis; bismuth nitrate

\section{Introduction}

In multicomponent reactions (MCRs), more than two reactants combine in a sequential manner to produce in a highly selective fashion a product that retains the majority of the atoms of the starting materials. In a MCR, a product is assembled according to a cascade of elementary chemical reactions. Thus, there is a network of reaction equilibria, which all finally flow into an irreversible step yielding 
the final product. Such processes are of great interest in diversity-oriented synthesis, especially to generate compounds libraries for screening purposes [1-6]. The Hantzsch reaction [7], the oldest known synthesis of 1,4-dihydropyridines (1,4-DHPs) and their derivatives, is one of the most general methods (changed) useful for the synthesis of a number of medicinally and pharmacologically molecules which includes commercial drugs such as nifedipine [8], felodipine [9], nicardipine [10] and amlodipine [11] which are used in treatment of angina and hypertension. It has been reported [12-14] that the pharmaceutical action of these drugs is related to binding to voltage dependent L-type of calcium channel and thus decreasing the passage of $\mathrm{Ca}^{2+}$ ions to the cell. The result is relaxation of smooth muscle cells and lowering of the blood pressure. An alternate mechanism on a molecular level has been found and is based on increased NO release from the intact endothelium [15]. In addition, 1,4-dihydropydines have been reported as anticancer [16], neurotropic [17], glycoprotein inhibitors [18], bronchodilating [19], and antidiabetic [20] agents.

Recently a number of publications have appeared on the synthesis of 1,4-dihydropyridines [21-27]. Nonetheless, this reaction is still under active investigation because of the importance of the dihydropyridine products. A number of new catalysts, solid supports and solvents have been used for this reaction. Some recent examples include molecular iodine [28], ruthenium trichloride [29], lithium bromide [30], $\mathrm{Zn}[(\mathrm{L}) \text { proline }]_{2}$ [31], nano aluminium nitride [32], bakers' yeast [33], Wells-Dawson heteropolyacid $\left(\mathrm{H}_{6} \mathrm{P}_{2} \mathrm{~W}_{18} \mathrm{O}_{62} \cdot 24 \mathrm{H}_{2} \mathrm{O}\right)$ [34], silica gel supported sodium bisulfate [35], solvents like trifluoroethanol [36], and ionic liquid [37]. Many of these reported methods involve the use of expensive reagents, hazardous solvents, long reaction times and tedious workup procedures. Therefore, it is desirable to develop a rapid, efficient and practical method for the synthesis of 1,4-dihydropyridines under eco-friendly conditions.

We report herein an easy and extremely rapid method for the preparation 1,4-dihydropyridines under solvent-free conditions in the presence of catalytic amounts of bismuth nitrate under microwave irradiation. In contrast to the existing methods, our method is extremely rapid, simple and high yielding (Scheme 1).

Scheme 1. Microwave-assisted one-pot, three component synthesis of 1,4-dihydropyridines using bismuth nitrate as catalyst (5 mol\%) under solventless condition.

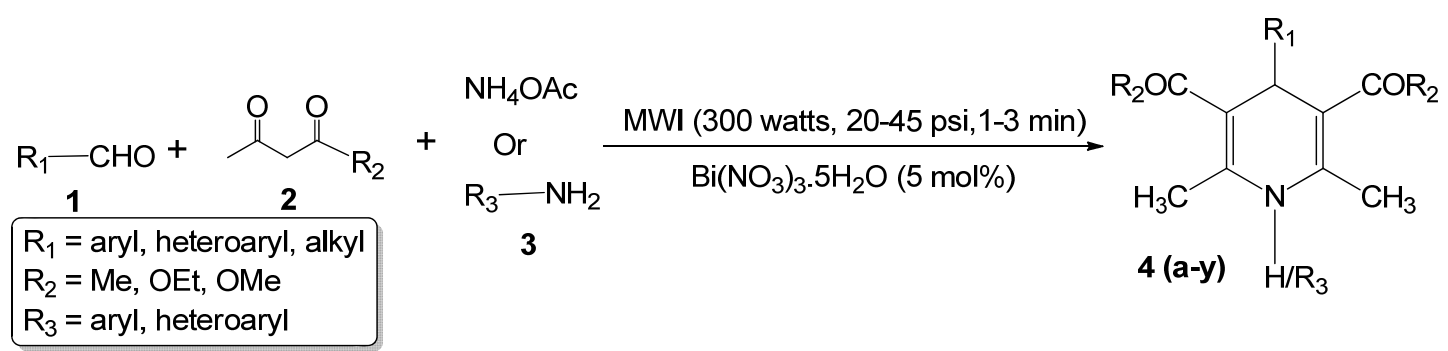

\section{Results and Discussion}

\subsection{Results}

We have been engaged in the study of microwave-induced reactions for many years. Using microwave irradiation technique we have successfully developed several new methodologies which 
include stereoselective synthesis of $\beta$-lactams [38-40], synthesis of pyrroles [41-44], aza-Michael addition [45], synthesis of quinoxalines [46], etc. On the other hand, organo-bismuth chemistry [47-50] is considered an emerging field of research in synthetic organic chemistry. We have demonstrated the catalytic activity of trivalent bismuth nitrate pentahydrate in a number of examples. These experiments resulted in various methods that include nitration of aromatic systems [51-53], Michael reactions [54], protection of carbonyl compounds [55], deprotection of oximes and hydrazones [56], Paal-Knorr synthesis of pyrroles [57], hydrolysis of amides [58], electrophilic substitution of indoles [59,60], synthesis of $\alpha$-aminophosphonates [61], and Biginelli condensation [62]. During the course of present study, it has been conceived that 1,4-dihydropyridine derivatives can be easily prepared using bismuth nitrate as the catalyst. Our success in the bismuth nitrate-induced reaction has revealed that this reagent acts as a Lewis acid. Moreover, it has been discovered that this reagent is compatible with the presence of sensitive functional groups. This idea has been extended in this paper to the reaction on carbonyl compounds (both aldehydes and 1,3-dicarbomyl compounds) with a suitable ammonia source in the presence of catalytic amounts of bismuth nitrate under solvent-free conditions.

Our initial work started with screening of catalyst loading and solvent so as to identify optimal reaction conditions for the synthesis of 1,4-dihydropyridines. First of all, a number of bismuth salts, e.g., bismuth chloride, bismuth triflate, bismuth subnitrate, bismuth bromide, bismuth iodide and bismuth nitrate pentahydrate have been screened using benzaldehyde, ethyl acetoacetate and ammonium acetate as a model reaction under automated CEM microwave irradiation conditions $\left(300 \mathrm{Watts}, 50{ }^{\circ} \mathrm{C}\right.$, $1 \mathrm{~min})$. The results are shown in Table 1.

Table 1. Microwave-assisted one-pot, three component synthesis of 1,4-dihydropyridines from benzaldehyde $(1 \mathrm{mmol})$, ethyl acetoacetate $(2 \mathrm{mmol})$ and ammonium acetate (1 mmol) using bismuth-salts as catalyst $(10 \mathrm{~mol} \%)$ under solventless condition $(1 \mathrm{~min})$ : catalyst optimization.

\begin{tabular}{lll}
\hline Entry & Bi-salt (10 mol\%) & Yield (\%) \\
\hline 1 & $\mathrm{BiCl}_{3}$ & 81 \\
2 & $\mathrm{Bi}(\mathrm{OTf})_{3}$ & 79 \\
3 & $\mathrm{BiI}_{3}$ & 72 \\
4 & $\mathrm{Bi}_{5} \mathrm{O}(\mathrm{OH})_{9}\left(\mathrm{NO}_{3}\right)_{3}$ & 67 \\
5 & $\mathrm{BiBr}_{3}$ & 73 \\
6 & $\mathrm{Bi}\left(\mathrm{NO}_{3}\right)_{3} .5 \mathrm{H}_{2} \mathrm{O}$ & 96 \\
7 & $\mathrm{No} \mathrm{catalyst}$ & 52 \\
\hline \multicolumn{2}{c}{ a isolated yield. }
\end{tabular}

Bismuth nitrate pentahydrate was found to be the best catalyst under these conditions (Entry 6, Table 1). The reaction proceded in the absence of any catalyst (only microwave irradiation) in $52 \%$ yield within a minute (Entry 7, Table 1). When continued for 10 minutes the yield of the desired product could be increased to $64 \%$. The same reaction was used to optimize the amount of the catalyst (Table 2). The results show that $5 \mathrm{~mol} \%$ bismuth nitrate pentahydrate is required to complete the reaction within one minute (Entry 6, Table 2). The reaction was then performed in various solvents as well as under neat conditions under identical microwave power using bismuth nitrate pentahydrate $(5 \mathrm{~mol} \%)$ as the catalyst to identify the best conditions (Table 3). The results suggest that bismuth nitrate pentahydrate 
is the best catalyst under neat conditions for the reaction (Entry 8, Table 3). Considering the above observations we next carried out a series of reactions using various aldehydes, carbonyl compounds and aromatic amines/ammonium acetate in presence of bismuth nitrate pentahydrate (5 mol\%) under microwave irradiation.

Table 2. Microwave-assisted one-pot, three component synthesis of 1,4-dihydropyridines from benzaldehyde $(1 \mathrm{mmol})$, ethyl acetoacetate $(2 \mathrm{mmol})$ and ammonium acetate (1 mmol) using bismuth nitrate pentahydrate as catalyst under solventless condition (1 minute): optimization of the amount of the catalyst.

\begin{tabular}{ccc}
\hline Entry & $\mathbf{B i}\left(\mathbf{N O}_{\mathbf{3}}\right)_{\mathbf{3}} \mathbf{. 5} \mathbf{H}_{\mathbf{2}} \mathbf{O}(\mathbf{m o l} \mathbf{\%})$ & Yield (\%) $^{\mathbf{a}}$ \\
\hline 1 & 30 & 88 \\
2 & 25 & 87 \\
3 & 20 & 93 \\
4 & 15 & 91 \\
5 & 10 & 96 \\
6 & 5 & 99 \\
7 & 2 & 86 \\
8 & 1 & 73 \\
\hline \multicolumn{3}{c}{}
\end{tabular}

Table 3. Microwave-assisted one-pot, three component synthesis of 1,4-dihydropyridines from benzaldehyde $(1 \mathrm{mmol})$, ethyl acetoacetate $(2 \mathrm{mmol})$ and ammonium acetate $(1 \mathrm{mmol})$ using bismuth nitrate pentahydrate as catalyst (5 mol\%) for 1 minute: Solvent optimization.

\begin{tabular}{lll}
\hline Entry & Solvent (1 mL) & Yield (\%) $^{\text {a }}$ \\
\hline 1 & Water & 91 \\
2 & THF & 77 \\
3 & Ethanol & 79 \\
4 & Toluene & 62 \\
5 & Methanol & 76 \\
6 & Dichloromethane & 71 \\
7 & DMSO & 76 \\
8 & Neat & 99 \\
\hline \multicolumn{3}{c}{${ }^{a}$ isolated yield. }
\end{tabular}

Bismuth nitrate pentahydrate, a commercially available solid salt, is very economical and much less toxic than other Lewis acids. It is very convenient to conduct reactions with bismuth nitrate because of its stability in the presence of moisture and oxygen. In all the cases, the reactions were completed within 1-3 min and the products were obtained in excellent yield (Table 4).

\subsection{Discussion}

A series of 1,4-dihydropyridines were synthesized by using diverse aldehydes, 1,3-diketo compounds and ammonium acetate/amines in the presence of bismuth nitrate pentahydrate $(5 \mathrm{~mol} \%)$ as catalyst under microwave irradiation. As shown in Table 4, the reaction proceeded equally well 
irrespective of the nature of the carbonyl compounds (aliphatic, aromatic, heteroaromatic) or amines (aromatic, heteroaromatic) to afford the corresponding products in excellent yield (87\%-99\%). The catalytic system worked well with acid-sensitive heteroaromatic aldehyde (Entries 17-22, Table 4), $\alpha$, $\beta$-unsaturated aldehyde (Entry 23, Table 4) and aliphatic aldehydes (Entries 24-25, Table 4). Aromatic primary amines (Entries 4, 6, 8, 9, 14, 20 and 22, Table 4) and heterocyclic amines (Entries 5 and 21, Table 4) upon reaction with aldehydes and 1,3-diketo compounds produced the corresponding products in excellent yields. When ammonium acetate was used as ammonia source (Entries 1-3, 7, 10-13, 15-19, 23-25, Table 4) equally excellent yields of the corresponding products were isolated. Importantly, all reactions were completed within 1-3 min (Table 4). Tajbakhsh et al. reported [63] a bismuth nitrateinduced oxidation of Hantzsch 1,4-dihydropyridines in presence of solid support (silica gel) under microwave (kitchen) irradiation. However, such oxidation was completely avoided using controlled microwave irradiation ( 300 Watts, $50{ }^{\circ} \mathrm{C}, 24-45 \mathrm{psi}$ ) under neat conditions (no solid support or solvent).

Table 4. Microwave-assisted one-pot, three component synthesis of 1,4-dihydropyridines using bismuth nitrate as catalyst ( $5 \mathrm{~mol} \%$ ) under solventless condition following Scheme 1.

\begin{tabular}{|c|c|c|c|c|c|c|}
\hline Entry & Aldehyde & $\begin{array}{c}1,3- \\
\text { Dicarbonyl } \\
\text { compounds }\end{array}$ & $\begin{array}{c}\text { Ammonia } \\
\text { source }\end{array}$ & Product & $\begin{array}{l}\text { Time } \\
(\min )\end{array}$ & $\begin{array}{l}\text { Yield } \\
(\%)^{a}\end{array}$ \\
\hline 1 & & & $\mathrm{NH}_{4} \mathrm{OAc}$ & & 1 & 99 \\
\hline 2 & & & $\mathrm{NH}_{4} \mathrm{OAc}$ & & 2.5 & 91 \\
\hline 3 & & & $\mathrm{NH}_{4} \mathrm{OAc}$ & & 2 & 93 \\
\hline 4 & & & & & 1.5 & 94 \\
\hline
\end{tabular}


Table 4. Cont.

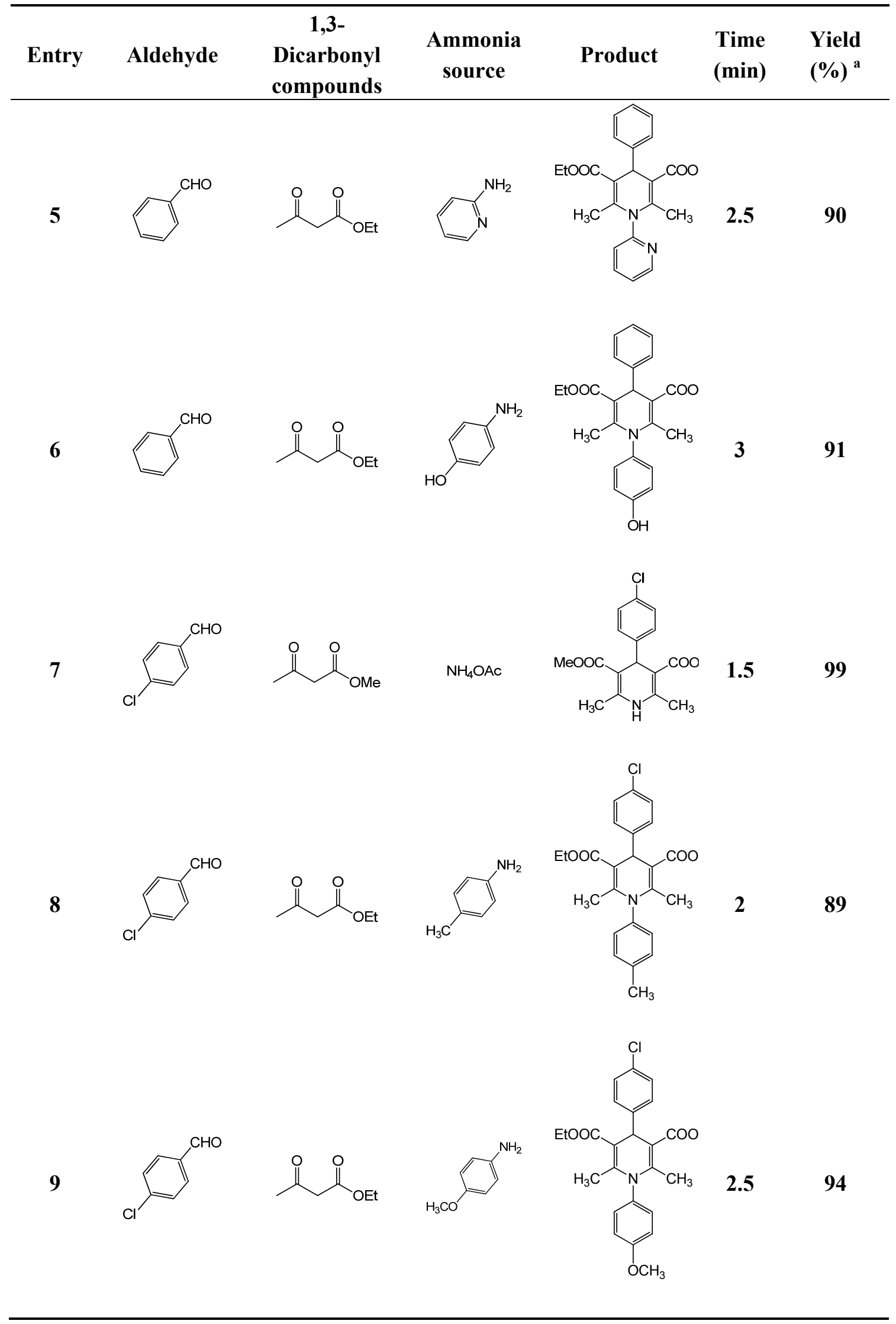


Table 4. Cont.

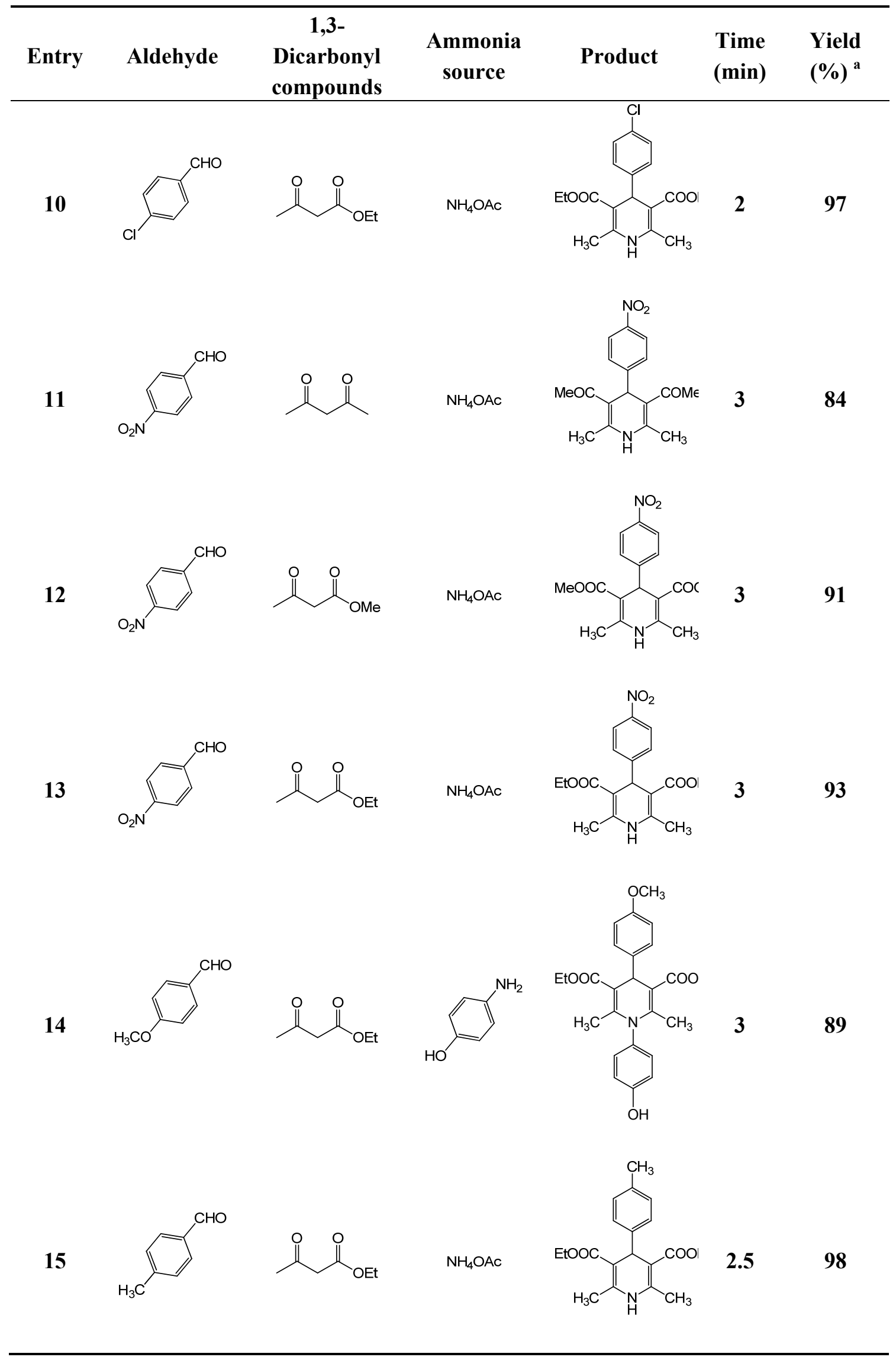


Table 4. Cont.

\begin{tabular}{|c|c|c|c|c|c|c|}
\hline Entry & Aldehyde & $\begin{array}{c}1,3- \\
\text { Dicarbonyl } \\
\text { compounds }\end{array}$ & $\begin{array}{c}\text { Ammonia } \\
\text { source }\end{array}$ & Product & $\begin{array}{l}\text { Time } \\
\text { (min) }\end{array}$ & $\begin{array}{l}\text { Yield } \\
(\%)^{a}\end{array}$ \\
\hline 16 & & & $\mathrm{NH}_{4} \mathrm{OAc}$ & & 3 & 95 \\
\hline 17 & & & $\mathrm{NH}_{4} \mathrm{OAc}$ & & 2 & 95 \\
\hline 18 & & & $\mathrm{NH}_{4} \mathrm{OAc}$ & & 2 & 93 \\
\hline 19 & & & $\mathrm{NH}_{4} \mathrm{OAc}$ & & 2 & 98 \\
\hline 20 & & & & & 2.5 & 97 \\
\hline 21 & & & & & 3 & 90 \\
\hline
\end{tabular}


Table 4. Cont.

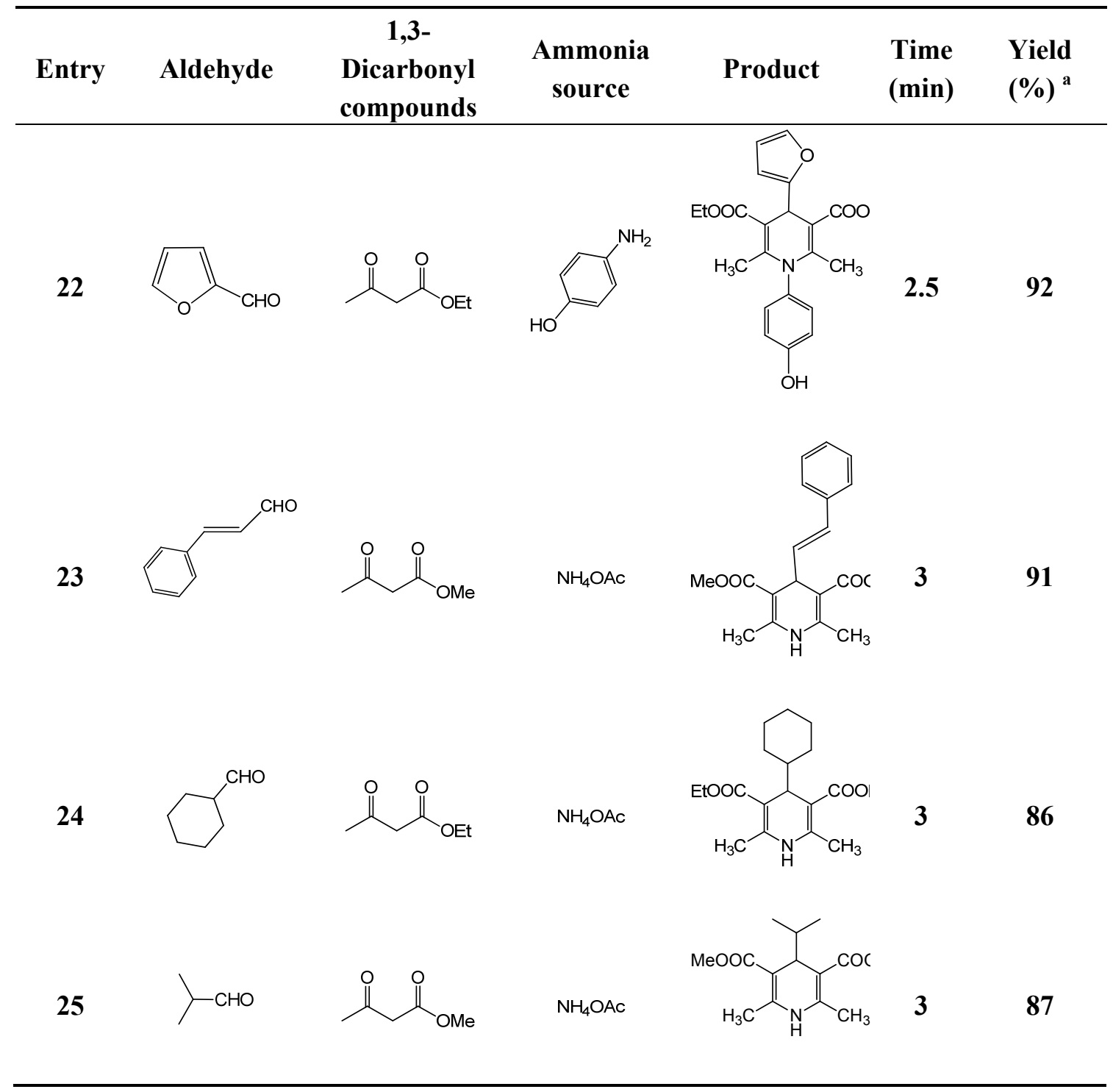

${ }^{\mathrm{a}}$ isolated yield.

The selectivity observed in our current method for the preparation of 1,4-dihydropyridines is interesting because further oxidation to pyridine derivatives could be prevented under control of the exposure to microwave irradiation and judicial choice of the catalyst. Although we have had success in the preparation of 1,4-dihydropyridines following the above method, however, the mechanism has yet to be established. We have been working on the synthesis and biological evaluation of various $\beta$-lactams as novel anticancer agents [64-70] over the past several years. The synthesis of $\beta$-lactams through imines requires a carbonyl compound and an amine. Our study in this field, suggests that carbonyl compounds, amines/ammonium acetate and 1,3-dicarbonyl compounds in the presence of Lewis acid will produce 1,4-dihydropyridines in excellent yield. This hypothesis has been tested by reacting several carbonyl compounds and 1,3-dicarbonyl compounds using aromatic amine/ammonium acetate as ammonia source in the presence of catalytic amount of bismuth nitrate pentahydrate (Table 4) following Scheme 1. Although the mechanism of the reaction has not been studied in detail, based on the structure of the starting materials and products, a plausible mechanistic pathway may be suggested as follows. 
In the initial step, bismuth nitrate acts as Lewis acid to facilitate the formation of the corresponding imine through a condensation of the amine/ammonium acetate and aldehyde. The 1,3-diketo compound is also activated due to the presence of bismuth nitrate and the equilibrium is shifted toward the enol form. As a result, a nucleophilic attack to imine carbon can take place. A second nucleophilic attack by another enol tautomer to the same carbon, subsequent ring-closure and dehydration yield the product (Scheme 2). Microwaves, as a part of electromagnetic spectrum, are composed of two field components: electric and the magnetic. For the purpose of heating, the electric component is important as it results in a force being applied to all the polar or charged species. Such species, in response to the electric field, start to move or rotate and this causes additional polarization of the polar species in the vicinity. When dipolar species are subjected to the electric component of microwave fields they start to oscillate, following the oscillation of the electric field. During such oscillation, the polar or charged species collide with neighboring particles (charged or neutral). This rapid motion and resulting intermolecular friction cause an intense internal heat that can increase the rate of reaction [71]. It is obvious that the dielectric properties of the material under consideration are of paramount importance. In presence of microwaves, bismuth nitrate increases the "anionic activation" [72]. On this basis, the relative permittivity (ability of a molecule to be polarized by the application of an electric field) of the carbonyl groups (aldehyde as well as the diketo compound) (Scheme 2) increases, which facilitates microwave heating extensively. The larger the relative permittivity of a substance, the greater will be the coupling with microwaves [73]. When the reagents (amine, aldehyde and 1,3-diketo compound) and the catalyst (bismuth nitrate pentahydrate) are subjected to microwave irradiation, microwaves passes through the (glass) walls of the reaction vessel and heat only the reactants avoiding local overheating at the reaction walls. This can eliminate reaction side products and helps to explain the higher yields and purities. The extreme rapidity with excellent yield of the reaction can be rationalized as a synergistic effect of the Lewis acid catalyst (bismuth nitrate) and microwave irradiation.

Scheme 2. Plausible mechanistic pathway for the synthesis of 1,4-dihydropyridines.
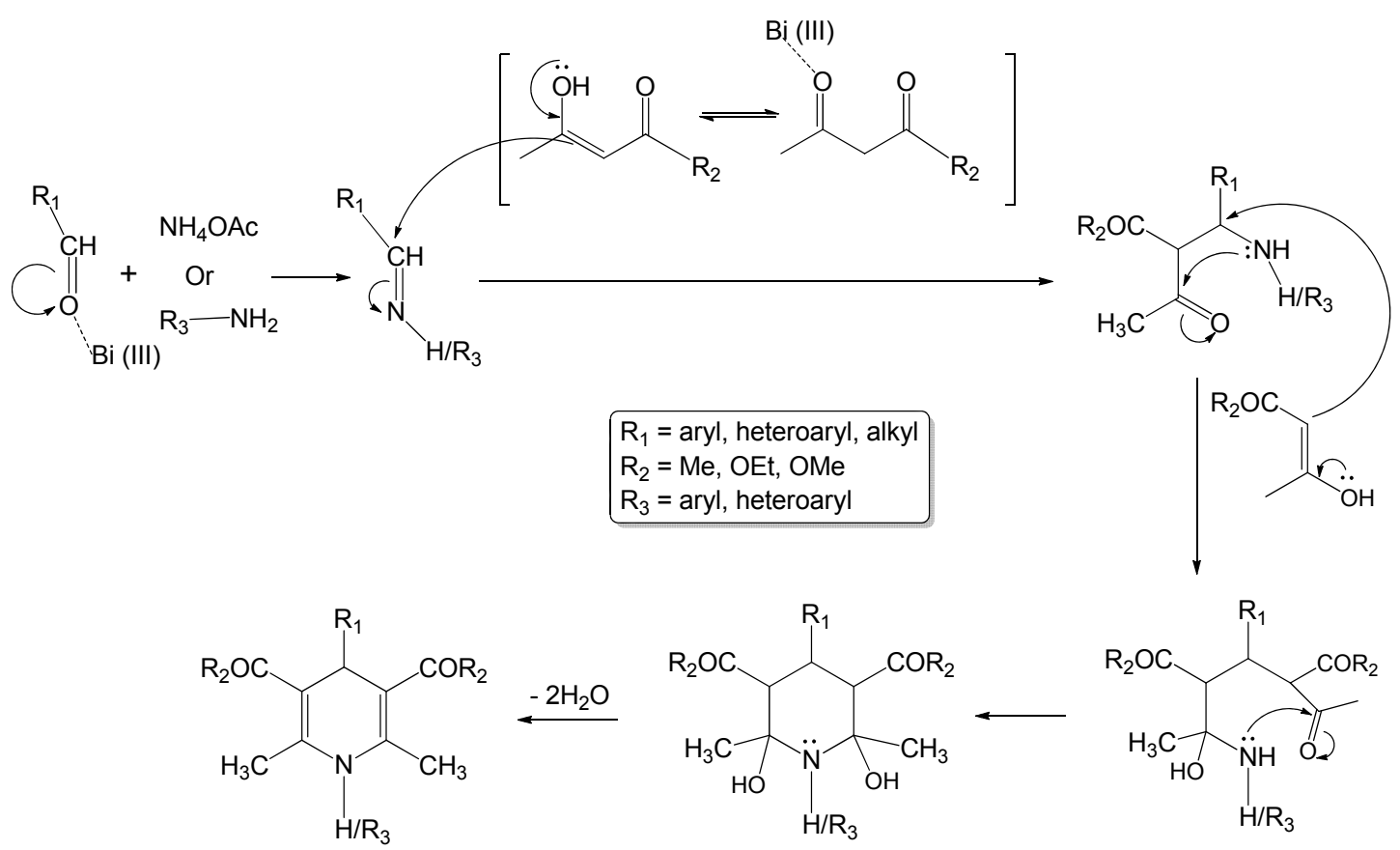


\section{Experimental}

\subsection{General}

Melting points were determined in a Fisher Scientific electrochemical Mel-Temp* manual melting point apparatus (Model 1001) equipped with a $300{ }^{\circ} \mathrm{C}$ thermometer. FT-IR spectra were registered on a Bruker IFS 55 Equinox FTIR spectrophotometer as $\mathrm{KBr}$ discs. ${ }^{1} \mathrm{H}-\mathrm{NMR}$ (600 MHz) and ${ }^{13} \mathrm{C}-\mathrm{NMR}$ $(150 \mathrm{MHz})$ spectra were obtained at room temperature with Bruker superconducting Ultrashield ${ }^{\mathrm{TM}}$ Plus $600 \mathrm{MHz}$ NMR spectrometer with central field 14.09 Tesla, coil inductance 89.1 Henry and magnetic energy $1127.2 \mathrm{~kJ}$ using $\mathrm{CDCl}_{3}$ as solvent. Elemental analyses $(\mathrm{C}, \mathrm{H}, \mathrm{N})$ were conducted using the Perkin-Elmer 2400 series II elemental analyzer, their results were found to be in good agreement $( \pm 0.2 \%)$ with the calculated values for $\mathrm{C}, \mathrm{H}, \mathrm{N}$. Bismuth nitrate pentahydrate (reagent grade) 98\% (Cat \# 248592-500G, Batch \# MKBC6772) purchased from Sigma-Aldrich Corporation was used. All other chemicals were purchased from Sigma-Aldrich Corporation (analytical grade). Throughout the project solvents were purchased from Fisher-Scientific. Deionized water was used for the preparation of all aqueous solutions.

\subsection{General Procedure for the One-Pot, Three-Component Synthesis of 1,4-Dihydropyridines}

A representative experimental procedure (Entry 1, Table 4) is as follows: bismuth nitrate pentahydrate $(5 \mathrm{~mol} \%, 24 \mathrm{mg}$ ) was added to a mixture of ammonium acetate ( $1 \mathrm{mmol}, 77 \mathrm{mg})$, benzaldehyde $(1 \mathrm{mmol}, 0.1 \mathrm{~mL})$ and ethyl acetoacetate $(2 \mathrm{mmol}, 0.25 \mathrm{~mL})$ in a microwave vial equipped with a small magnetic stir bar. The resulting mixture was stirred under automated CEM microwave irradiation at $50{ }^{\circ} \mathrm{C}(300$ Watts, $20-45 \mathrm{psi})$ and the progress of the reaction was monitored by TLC every 30 seconds. After completion of the reaction (Table 4$)$ ethyl acetate $(5 \mathrm{~mL})$ was added and the solution was filtered and washed successively with brine $(3 \mathrm{~mL})$ and water $(3 \mathrm{~mL})$. It was dried over anhydrous sodium sulphate and filtered. A short column of silica gel was used to isolate the product 2,6-dimethyl-4-phenyl-1,4-dihydropyridine-3,5-diethylcarboxylate in 99\% overall yield.

2,6-Dimethyl-4-phenyl-1,4-dihydropyridine-3,5-diethylcarboxylate (Entry 1, Table 4). White solid; mp 158-159 ${ }^{\circ} \mathrm{C}$; IR (KBr) 3320, 1698, 1653, 1480, 1215, $790 \mathrm{~cm}^{-1} ;{ }^{1} \mathrm{H}-\mathrm{NMR}\left(\mathrm{CDCl}_{3}\right) \delta 1.23(\mathrm{t}, J=7.0$, 6H), 2.34 (s, 3H), 2.33 (s, 3H), 4.09 (q, J=7.2 Hz, 4H), 4.99 (s, 1H), 5.96 (br s, 1H, NH), 7.09-7.34 $(\mathrm{m}, 5 \mathrm{H}) ;{ }^{13} \mathrm{C}-\mathrm{NMR}\left(\mathrm{CDCl}_{3}\right) \delta 14.08,19.43,39.46,59.41,102.89,127.34,129.76,136.32,145.66$, 147.10, 165.09. Anal. Calcd for $\mathrm{C}_{19} \mathrm{H}_{23} \mathrm{NO}_{4}$ : C, 69.28; H, 7.04, N, 4.25. Found: C, 69.05; H, 6.98; N, 4.29.

2,6-Dimethyl-4-phenyl-1,4-dihydropyridine-3,5-diethanone (Entry 2, Table 4). Yellowish white solid; mp 134-137 ${ }^{\circ} \mathrm{C}$; IR (KBr) 3362, 3078, 1721, $612 \mathrm{~cm}^{-1}$; ${ }^{1} \mathrm{H}-\mathrm{NMR}\left(\mathrm{CDCl}_{3}\right) \delta 2.21(\mathrm{~s}, 6 \mathrm{H}), 2.24(\mathrm{~s}, 6 \mathrm{H})$, $4.90(\mathrm{~s}, 1 \mathrm{H}), 6.02(\mathrm{br} \mathrm{s}, 1 \mathrm{H}, \mathrm{NH}), 7.22-7.36(\mathrm{~m}, 5 \mathrm{H}) ;{ }^{13} \mathrm{C}-\mathrm{NMR}\left(\mathrm{CDCl}_{3}\right) \delta 22.06,29.61,36.87,115.67$, 123.89, 126.32, 129.55, 142.56, 147.11, 197.01. Anal. Calcd for $\mathrm{C}_{17} \mathrm{H}_{19} \mathrm{NO}_{2}: \mathrm{C}, 75.81 ; \mathrm{H}, 7.11, \mathrm{~N}$, 5.20. Found: C, 75.71; H, 7.14; N, 5.11.

2,6-Dimethyl-4-phenyl-1,4-dihydropyridine-3,5-dimethylcarboxylate (Entry 3, Table 4). White solid; mp 116-118 ${ }^{\circ} \mathrm{C}$; IR (KBr) cm ${ }^{-1}$; 3362, 3065, 2983, 1705, 1112; ${ }^{1} \mathrm{H}-\mathrm{NMR}\left(\mathrm{CDCl}_{3}\right) \delta 2.27(\mathrm{~s}, 6 \mathrm{H}), 3.74$ $(\mathrm{s}, 6 \mathrm{H}), 4.84(\mathrm{~s}, 1 \mathrm{H}), 7.23-7.34(\mathrm{~m}, 5 \mathrm{H}) ;{ }^{13} \mathrm{C}-\mathrm{NMR}\left(\mathrm{CDCl}_{3}\right) \delta$ 20.09, 41.09, 55.77, 105.84, 128.51, 
129.08, 131.92, 143.58, 149.43, 166.59. Anal. Calcd for $\mathrm{C}_{17} \mathrm{H}_{19} \mathrm{NO}_{4}$ : C, 67.76; H, 6.36, N, 4.65. Found: C, 67.65; H, 6.29; N, 4.62 .

2,6-Dimethyl-1,4-diphenyl-1,4-dihydropyridine-3,5-diethylcarboxylate (Entry 4, Table 4). Brownish white solid; mp 161-162 ${ }^{\circ} \mathrm{C}$; IR (KBr) 3325, 2987, 1722, 1624, $1092 \mathrm{~cm}^{-1} ;{ }^{1} \mathrm{H}-\mathrm{NMR}\left(\mathrm{CDCl}_{3}\right) \delta 1.19$ $(\mathrm{t}, J=7.02,6 \mathrm{H}), 2.29(\mathrm{~s}, 6 \mathrm{H}), 4.18(\mathrm{q}, J=6.3 \mathrm{~Hz}, 4 \mathrm{H}), 4.99(\mathrm{~s}, 1 \mathrm{H}), 7.30-7.56(\mathrm{~m}, 10 \mathrm{H}) ;{ }^{13} \mathrm{C}-\mathrm{NMR}$ $\left(\mathrm{CDCl}_{3}\right) \delta 16.02,19.76,44.34,62.87,105.39,122.32,123.45,126.77,127.47,128.43,130.58,141.55$, 144.39, 153.84, 167.09. Anal. Calcd for $\mathrm{C}_{15} \mathrm{H}_{27} \mathrm{NO}_{4}$ : C, 74.05; H, 6.67, N, 3.45. Found: C, 74.11; $\mathrm{H}$, $6.72 ; \mathrm{N}, 3.41$.

2,6-Dimethyl-4-phenyl-1,4-dihydropyridine-1-(2'-pyridyl)-3,5-diethylcarboxylate (Entry 5, Table 4). White solid; mp $144-146{ }^{\circ} \mathrm{C}$; IR (KBr) 3110, 2961, 1692, 1633, $1041 \mathrm{~cm}^{-1}$; ${ }^{1} \mathrm{H}-\mathrm{NMR}\left(\mathrm{d}_{6}\right.$-DMSO) $\delta$ $1.21(\mathrm{t}, J=7.1 \mathrm{~Hz}, 6 \mathrm{H}), 2.24(\mathrm{~s}, 6 \mathrm{H}), 4.21(\mathrm{q}, J=7.2 \mathrm{~Hz}, 4 \mathrm{H}), 4.82(\mathrm{~s}, 1 \mathrm{H}), 6.89-8.11(\mathrm{~m}, 9 \mathrm{H})$; ${ }^{13} \mathrm{C}-\mathrm{NMR}\left(\mathrm{d}_{6}\right.$-DMSO) $\delta 15.01,19.46,45.71,63.82,104.62,115.48,124.42,124.61,127.91,129.59$, 139.62, 145.85, 148.29, 150.73, 167.57. Anal. Calcd for $\mathrm{C}_{24} \mathrm{H}_{26} \mathrm{~N}_{2} \mathrm{O}_{4}: \mathrm{C}, 70.93 ; \mathrm{H}, 6.40, \mathrm{~N}, 6.89$. Found: C, 70.87; H, 6.41; N, 6.90 .

2,6-Dimethyl-1-(4-hydroxyphenyl)-4-phenyl-1,4-dihydropyridine-3,5-diethylcarboxylate (Entry 6, Table 4). Yellow solid; mp 90-91 ${ }^{\circ} \mathrm{C}$; IR (KBr) 3433, 3055, 2910, 1712, 1698, 1482, $790 \mathrm{~cm}^{-1}$; ${ }^{1} \mathrm{H}-\mathrm{NMR}\left(\mathrm{CDCl}_{3}\right) \delta 1.22(\mathrm{t}, J=7.2,6 \mathrm{H}), 2.29(\mathrm{~s}, 6 \mathrm{H}), 4.14(\mathrm{q}, 4 \mathrm{H}), 5.11(\mathrm{~s}, 1 \mathrm{H}), 6.92-7.41(\mathrm{~m}, 9 \mathrm{H})$; ${ }^{13} \mathrm{C}-\mathrm{NMR}\left(\mathrm{CDCl}_{3}\right) \delta 14.91,20.54,42.52,62.41,104.11,117.34,122.64,125.29,127.47,128.76$, 134.32, 145.16, 148.51, 153.12, 167.03. Anal. Calcd for $\mathrm{C}_{25} \mathrm{H}_{27} \mathrm{NO}_{5}$ : C, 71.24; H, 6.46, N, 3.32 . Found: C, 70.98; H, 6.37; N, 3.38.

2,6-Dimethyl-4-(4-chlorophenyl)-1,4-dihydropyridine-3,5-dimethylcarboxylate (Entry 7, Table 4). Yellow solid; mp 194-195 ${ }^{\circ} \mathrm{C}$; IR (KBr) 3325, 1701, 1653, 1371, $1210 \mathrm{~cm}^{-1} ;{ }^{1} \mathrm{H}-\mathrm{NMR}\left(\mathrm{CDCl}_{3}\right) \delta 2.32$ (s, 6H), 3.69 (s, 6H), $4.93(\mathrm{~s}, 1 \mathrm{H}), 5.69$ (br. s, 1H), 7.20-7.27 (m, 4H); ${ }^{13} \mathrm{C}-\mathrm{NMR}\left(\mathrm{CDCl}_{3}\right) \delta 19.46$, 40.01, 52.00, 103.73, 128.14,129.32, 131.56, 144.65, 146.04, 167.89. Anal. Calcd for $\mathrm{C}_{17} \mathrm{H}_{18} \mathrm{ClNO}_{4}$ : C, 60.81; H, 5.40, N, 4.17. Found: C, 60.65; H, 5.43; N, 4.27.

2,6-Dimethyl-1-(4-methylphenyl)-4(4-chlorophenyl)-1,4-dihydropyridine-3,5-diethylcarboxylate (Entry 8, Table 4). Brown solid; mp 102-104 ${ }^{\circ} \mathrm{C}$; IR (KBr) 3312, 1705, 1681, 1290, $1140 \mathrm{~cm}^{-1}$; ${ }^{1} \mathrm{H}-\mathrm{NMR}$ $\left(\mathrm{CDCl}_{3}\right) \delta ; 1.22-1.26(\mathrm{~m}, 6 \mathrm{H}), 2.05(\mathrm{~s}, 6 \mathrm{H}), 2.41(\mathrm{~s}, 3 \mathrm{H}), 4.11(\mathrm{~m}, 4 \mathrm{H}), 5.01(\mathrm{~s}, 1 \mathrm{H}), 6.98-7.35(\mathrm{~m}, 8 \mathrm{H})$; ${ }^{13} \mathrm{C}-\mathrm{NMR}\left(\mathrm{CDCl}_{3}\right) \delta 15.63,20.92,22.30,45.76,61.51,105.73,124.72,128.64,128.99,130.02$, 131.84, 131.98, 139.41, 143.39, 155.91, 167.09. Anal. Calcd for $\mathrm{C}_{26} \mathrm{H}_{28} \mathrm{ClNO}_{4}: \mathrm{C}, 68.79 ; \mathrm{H}, 6.22, \mathrm{~N}$, 3.09. Found: C, 68.71; H, 6.27; N, 3.02 .

2,6-Dimethyl-1-(4-methoxyphenyl)-4-(4-chlorophenyl)-1,4-dihydropyridine-3,5-diethcarboxylate (Entry 9, Table 4). Brown solid; mp 134-136 ${ }^{\circ} \mathrm{C}$; IR (KBr) 3310, 1702, 1674, 1543, 1201, $972 \mathrm{~cm}^{-1}$; ${ }^{1} \mathrm{H}$ NMR $\left(\mathrm{CDCl}_{3}\right) \delta ; 1.23-1.25(\mathrm{~m}, 6 \mathrm{H}), 2.06(\mathrm{~s}, 6 \mathrm{H}), 3.85(\mathrm{~s}, 3 \mathrm{H}), 4.11-4.15(\mathrm{~m}, 4 \mathrm{H}), 5.09(\mathrm{~s}, 1 \mathrm{H}), 6.92-7.31$ $(\mathrm{m}, 8 \mathrm{H}) ;{ }^{13} \mathrm{C}-\mathrm{NMR}\left(\mathrm{CDCl}_{3}\right) \delta 15.87,21.49,45.75,55.75,61.54,104.48,118.58,122.74,127.99$, 130.07, 132.38, 134.29, 143.59, 154.20, 155.25, 167.21. Anal. Calcd for $\mathrm{C}_{26} \mathrm{H}_{28} \mathrm{ClNO}_{5}$ : C, 66.45; $\mathrm{H}$, 6.01, N, 2.98. Found: C, 66.37; H, 5.91; N, 2.93. 
2,6-Dimethyl-4-(4-chlorophenyl)-1,4-dihydropyridine-3,5-diethylcarboxylate (Entry 10, Table 4). White solid; mp $145-146^{\circ} \mathrm{C}$; IR (KBr) 3357, 1697, 1654, 1490, 1210, 1122, 790, $672 \mathrm{~cm}^{-1}$; ${ }^{1} \mathrm{H}-\mathrm{NMR}$ $\left(\mathrm{CDCl}_{3}\right) \delta 1.24(\mathrm{t}, J=7.2 \mathrm{~Hz}, 6 \mathrm{H}), 2.34(\mathrm{~s}, 6 \mathrm{H}), 4.10(\mathrm{q}, J=7.2 \mathrm{~Hz}, 4 \mathrm{H}), 4.96(\mathrm{~s}, 1 \mathrm{H}), 5.89$ (br s, 1H), 7.20-7.24 (m, 4H); ${ }^{13} \mathrm{C}-\mathrm{NMR}\left(\mathrm{CDCl}_{3}\right) \delta 14.39,19.61,39.22,59.90,103.79,127.90,129.41,131.73$, 144.12, 146.32, 167.53. Anal. Calcd for $\mathrm{C}_{19} \mathrm{H}_{22} \mathrm{ClNO}_{4}$ : C, 62.72; H, 6.09, N, 3.85. Found: C, 62.66; H, $6.00 ; \mathrm{N}, 3.81$.

2,6-Dimethyl-4-(4-nitrophenyl)-1,4-dihydropyridine-3,5-diethanone (Entry 11, Table 4). Yellow solid; mp 130-32 ${ }^{\circ} \mathrm{C}$; IR (KBr) 3372, 3066, 2980, 1720, 1492, 790, $712 \mathrm{~cm}^{-1} ;{ }^{1} \mathrm{H}-\mathrm{NMR}\left(\mathrm{CDCl}_{3}\right) \delta 2.27$ (s, $6 \mathrm{H}), 2.32$ (s, 6H), 4.72 (s, 1H), 5.94 (br s, 1H), 7.53 (d, $J=8.6 \mathrm{~Hz}, 2 \mathrm{H}), 8.13$ (d, $J=8.6 \mathrm{~Hz}, 2 \mathrm{H})$; ${ }^{13} \mathrm{C}-\mathrm{NMR}\left(\mathrm{CDCl}_{3}\right) \delta 20.76,27.18,34.14,111.08,124.53,127.51,143.83,147.27,151.20,195.26$. Anal. Calcd for $\mathrm{C}_{17} \mathrm{H}_{18} \mathrm{~N}_{2} \mathrm{O}_{4}$ : C, 64.96; H, 5.77, N, 8.91. Found: C, 64.90; H, 5.71; N, 9.01.

2,6-Dimethyl-4-(4-nitrophenyl)-1,4-dihydropyridine-3,5-dimethylcarboxylate (Entry 12, Table 4). Yellow solid; mp 152-154 ${ }^{\circ} \mathrm{C}$; IR ( $\left.\mathrm{KBr}\right) 3364,3092,2981,1698,1495,792 \mathrm{~cm}^{-1} ;{ }^{1} \mathrm{H}-\mathrm{NMR}\left(\mathrm{CDCl}_{3}\right) \delta$ $2.31(\mathrm{~s}, 6 \mathrm{H}), 3.75(\mathrm{~s}, 6 \mathrm{H}), 4.89(\mathrm{~s}, 1 \mathrm{H}), 5.91(\mathrm{br} \mathrm{s}, 1 \mathrm{H}), 7.52(\mathrm{~d}, J=8.6 \mathrm{~Hz}, 2 \mathrm{H}), 8.11(\mathrm{~d}, J=8.6 \mathrm{~Hz}$, $2 \mathrm{H}) ;{ }^{13} \mathrm{C}-\mathrm{NMR}\left(\mathrm{CDCl}_{3}\right) \delta 21.28,45.01,55.37,105.41,124.94,127.40,142.48,148.03,149.73,167.09$. Anal. Calcd for $\mathrm{C}_{17} \mathrm{H}_{18} \mathrm{~N}_{2} \mathrm{O}_{6}$ : C, 58.96; H, 5.24, N, 8.09. Found: C, 58.87; H, 5.16; N, 8.00.

2,6-Dimethyl-4-(4-nitrophenyl)-1,4-dihydropyridine-3,5-diethylcarboxylate (Entry 13, Table 4). Yellowish brown solid; mp $132-134{ }^{\circ} \mathrm{C}$; IR (KBr) 3316, 1705, 1649, 1521, 1215, 1120, $692 \mathrm{~cm}^{-1}$; ${ }^{1} \mathrm{H}-\mathrm{NMR}\left(\mathrm{CDCl}_{3}\right) \delta 1.22(\mathrm{t}, J=7.2 \mathrm{~Hz}, 6 \mathrm{H}), 2.34(\mathrm{~s}, 6 \mathrm{H}), 4.11(\mathrm{q}, J=7.1 \mathrm{~Hz}, 4 \mathrm{H}), 5.10(\mathrm{~s}, 1 \mathrm{H}), 6.15$ (br s, $1 \mathrm{H}), 7.51(\mathrm{~d}, J=8.6 \mathrm{~Hz}, 2 \mathrm{H}), 8.14(\mathrm{~d}, J=8.6 \mathrm{~Hz}, 2 \mathrm{H}) ;{ }^{13} \mathrm{C}-\mathrm{NMR}\left(\mathrm{CDCl}_{3}\right) \delta 14.19,19.53,40.11$, 60.34, 103.04, 123.63, 128.89, 145.04, 146.23, 155.21, 167.18. Anal. Calcd for $\mathrm{C}_{19} \mathrm{H}_{22} \mathrm{~N}_{2} \mathrm{O}_{6}: \mathrm{C}, 60.95$; H, 5.92, N, 7.48. Found: C, 60.87; H, 5.90; N, 7.41.

2,6-Dimethyl-1-(4-hydroxyphenyl)-4-(4-methoxyphenyl)-1,4-dihydropyridine-3,5-diethylcarboxylate (Entry 14, Table 4). Brown solid; mp 89-92 ${ }^{\circ} \mathrm{C}$; IR (KBr) 3435, 3046, 2927, 1718, 1639, $790 \mathrm{~cm}^{-1}$; ${ }^{1} \mathrm{H}-\mathrm{NMR}\left(\mathrm{CDCl}_{3}\right) \delta 1.25(\mathrm{t}, J=7.2 \mathrm{~Hz}, 6 \mathrm{H}), 2.21(\mathrm{~s}, 6 \mathrm{H}), 3.81(\mathrm{~s}, 3 \mathrm{H}), 4.03(\mathrm{q}, J=7.0 \mathrm{~Hz}, 4 \mathrm{H}), 5.22$ $(\mathrm{s}, 1 \mathrm{H}), 6.87-7.43(\mathrm{~m}, 8 \mathrm{H}), 9.43(\mathrm{~s}, 1 \mathrm{H}) ;{ }^{13} \mathrm{C}-\mathrm{NMR}\left(\mathrm{CDCl}_{3}\right) \delta 15.25,19.64,42.69,55.73,62.49$, 103.63, 115.84, 117.66, 123.08, 130.41, 134.53, 137.30, 147.59, 156.74, 167.12. Anal. Calcd for $\mathrm{C}_{26} \mathrm{H}_{29} \mathrm{NO}_{6}$ : C, 69.16; H, 6.47, N, 3.10. Found: C, 69.01; H, 6.37; N, 3.00.

2,6-Dimethyl-4-(4-methylphenyl)-1,4-dihydropyridine-3,5-diethylcarboxylate (Entry 15, Table 4). Yellow solid; mp $136-137{ }^{\circ} \mathrm{C}$; IR (KBr) 3342, 1693, 1648, 1492, 1210, 770, $612 \mathrm{~cm}^{-1}$; ${ }^{1} \mathrm{H}-\mathrm{NMR}$ $\left(\mathrm{CDCl}_{3}\right) \delta 1.27(\mathrm{t}, J=7.1 \mathrm{~Hz}, 6 \mathrm{H}), 2.29(\mathrm{~s}, 3 \mathrm{H}), 2.32(\mathrm{~s}, 6 \mathrm{H}), 4.13(\mathrm{q}, J=7.1 \mathrm{~Hz}, 4 \mathrm{H}), 4.97(\mathrm{~s}, 1 \mathrm{H})$, $6.10($ br s, $1 \mathrm{H}), 7.03(\mathrm{~d}, J=7.8 \mathrm{~Hz}, 2 \mathrm{H}), 7.23(\mathrm{~d}, J=7.8 \mathrm{~Hz}, 2 \mathrm{H}) ;{ }^{13} \mathrm{C}-\mathrm{NMR}\left(\mathrm{CDCl}_{3}\right) \delta$ 14.29, 19.42, $21.19,39.14,59.77,103.99,127.59,128.63,135.97,144.33,145.49,167.87$. Anal. Calcd for $\mathrm{C}_{20} \mathrm{H}_{25} \mathrm{NO}_{4}$ : C, 69.95; H, 7.34, N, 4.08. Found: C, 69.83; H, 7.23; N, 3.98 .

2,6-Dimethyl-4-(hydroxyphenyl)-1,4-dihydropyridine-3,5-diethylcarboxylate (Entry 16, Table 4). White solid; mp 233-234 ${ }^{\circ} \mathrm{C}$; IR (KBr) 3330, 1691, 1655, 1490, 1124, 790, $719 \mathrm{~cm}^{-1}$; ${ }^{1} \mathrm{H}-\mathrm{NMR}\left(\mathrm{CDCl}_{3}\right) \delta$ $1.24(\mathrm{t}, J=7.2 \mathrm{~Hz}, 6 \mathrm{H}), 2.33$ (s, 6H), 4.12 (q, $J=7.2 \mathrm{~Hz}, 4 \mathrm{H}), 5.30$ (s, 1H), 5.80 (br s, 1H), 6.66 (d, 
$J=8.9 \mathrm{~Hz}, 2 \mathrm{H}), 7.16(\mathrm{~d}, J=8.4 \mathrm{~Hz}, 2 \mathrm{H}) ;{ }^{13} \mathrm{C}-\mathrm{NMR}\left(\mathrm{CDCl}_{3}\right) \delta 14.20,19.84,39.88,59.73,103.81$, 127.83, 130.73, 130.38, 142.77, 148.52, 167.36. Anal. Calcd for $\mathrm{C}_{19} \mathrm{H}_{23} \mathrm{NO}_{5}: \mathrm{C}, 66.07 ; \mathrm{H}, 6.71, \mathrm{~N}$, 4.06. Found: C, 65.93; H, 6.59; N, 3.97.

2,6-Dimethyl-4-(2-furyl)-1,4-dihydropyridine-3,5-diethanone (Entry 3, Table 4). Yellowish white solid; mp 176-177 ${ }^{\circ} \mathrm{C}$; IR (KBr) 3361, 3077, 2985, 1722, 1495, $710 \mathrm{~cm}^{-1} ;{ }^{1} \mathrm{H}-\mathrm{NMR}\left(\mathrm{CDCl}_{3}\right) \delta 2.30$ $(\mathrm{s}, 6 \mathrm{H}), 2.33(\mathrm{~s}, 6 \mathrm{H}), 4.90(\mathrm{~s}, 1 \mathrm{H}), 5.89(\mathrm{br} \mathrm{s}, 1 \mathrm{H}), 6.13-6.21(\mathrm{~m}, 2 \mathrm{H}), 7.50(\mathrm{~m}, 1 \mathrm{H}) ;{ }^{13} \mathrm{C}-\mathrm{NMR}\left(\mathrm{CDCl}_{3}\right)$ $\delta$ 19.57, 27.04, 30.27, 107.52, 111.70, 114.21, 143.85, 149.59, 153.50, 197.11. Anal. Calcd for $\mathrm{C}_{15} \mathrm{H}_{17} \mathrm{NO}_{3}$ : C, 69.48; H, 6.61, N, 5.40. Found: C, 69.39; H, 6.52; N, 5.33.

2,6-Dimethyl-4-(2-furyl)-1,4-dihydropyridine-3,5-dimethylcarboxylate (Entry 18, Table 4). Yellow solid; mp 149-151 ${ }^{\circ} \mathrm{C}$; IR (KBr) 3352, 3080, 2992, 1795, 1743, 1460, 1110, $760 \mathrm{~cm}^{-1} ;{ }^{1} \mathrm{H}-\mathrm{NMR}\left(\mathrm{CDCl}_{3}\right) \delta$ $2.28(\mathrm{~s}, 6 \mathrm{H}), 3.76(\mathrm{~s}, 6 \mathrm{H}), 4.98(\mathrm{~s}, 1 \mathrm{H}), 5.90(\mathrm{br} \mathrm{s}, 1 \mathrm{H}), 6.13-6.21(\mathrm{~m}, 2 \mathrm{H}), 7.59(\mathrm{~m}, 1 \mathrm{H}) ;{ }^{13} \mathrm{C}-\mathrm{NMR}$ $\left(\mathrm{CDCl}_{3}\right) \delta 19.35,32.56,55.35,103.49,106.08,110.63,142.60,150.99,153.85,167.77$. Anal. Calcd for $\mathrm{C}_{15} \mathrm{H}_{17} \mathrm{NO}_{5}$ : C, 61.85; H, 5.88, N, 4.81. Found: C, 61.79; H, 5.81; N, 4.77.

2,6-Dimethyl-4-(2-furyl)-1,4-dihydropyridine-3,5-diethylcarboxylate (Entry 19, Table 4). Yellow solid; mp 161-162 ${ }^{\circ} \mathrm{C}$; IR (KBr) 3340, 1695, 1646, 1482, 1365, 1210, 1120, 750, $712 \mathrm{~cm}^{-1}$; ${ }^{1} \mathrm{H}-\mathrm{NMR}$ $\left(\mathrm{CDCl}_{3}\right) \delta 1.26(\mathrm{t}, J=7.1 \mathrm{~Hz}, 6 \mathrm{H}), 2.32(\mathrm{~s}, 6 \mathrm{H}), 4.18(\mathrm{q}, J=7.2 \mathrm{~Hz}, 4 \mathrm{H}), 5.21(\mathrm{~s}, 1 \mathrm{H}), 5.92($ br s, $1 \mathrm{H})$, 6.11-6.21 (m, 2H), $7.22(\mathrm{~m}, 1 \mathrm{H}) ;{ }^{13} \mathrm{C}-\mathrm{NMR}\left(\mathrm{CDCl}_{3}\right) \delta 14.29,19.44,33.32,59.89,101.38,104.51$, 110.03, 140.78, 145.55, 158.63, 167.61. Anal. Calcd for $\mathrm{C}_{17} \mathrm{H}_{21} \mathrm{NO}_{5}$ : C, 63.94; H, 6.63, N, 4.39. Found: C, 63.82; H, 6.51; N, 4.30 .

2,6-Dimethyl-1-(phenyl)-4-(2-furyl)-1,4-dihydropyridine-3,5-diethylcarboxylate (Entry 20, Table 4). Brown solid; mp 151-153 ${ }^{\circ} \mathrm{C}$; IR (KBr) 2985, 1671, 1630, 1065, 872, $740 \mathrm{~cm}^{-1} ;{ }^{1} \mathrm{H}-\mathrm{NMR}\left(\mathrm{CDCl}_{3}\right) \delta$ $1.23(\mathrm{t}, J=7.2 \mathrm{~Hz}, 6 \mathrm{H}), 2.21(\mathrm{~s}, 6 \mathrm{H}), 4.26(\mathrm{q}, J=7.0 \mathrm{~Hz}, 4 \mathrm{H}), 5.41(\mathrm{~s}, 1 \mathrm{H}), 6.11-6.20(\mathrm{~m}, 2 \mathrm{H}), 7.31-7.69$ $(\mathrm{m}, 6 \mathrm{H}) ;{ }^{13} \mathrm{C}-\mathrm{NMR}\left(\mathrm{CDCl}_{3}\right) \delta 15.66,18.87,34.18,62.33,103.00,105.42,111.04,122.04,123.11$, 128.40, 140.07, 143.77, 151.34, 154.39, 167.62. Anal. Calcd for $\mathrm{C}_{23} \mathrm{H}_{25} \mathrm{NO}_{5}: \mathrm{C}, 69.87 ; \mathrm{H}, 6.32, \mathrm{~N}$, 3.56. Found: C, 69.82; H, 6.28; N, 3.53 .

2,6-Dimethyl-1-(2'-pyridyl)-4-(2-furyl)-1,4-dihydropyridine-3,5-diethylcarboxylate (Entry 21, Table 4). White solid; mp 198-199 ${ }^{\circ} \mathrm{C}$; IR (KBr) 2990, 1693, 1636, 1410, 1035, $970 \mathrm{~cm}^{-1}$; ${ }^{1} \mathrm{H}-\mathrm{NMR}$ (d $\mathrm{d}_{6}$-DMSO) $\delta 1.24(\mathrm{t}, J=7.2 \mathrm{~Hz}, 6 \mathrm{H}), 2.39(\mathrm{~s}, 6 \mathrm{H}), 4.23(\mathrm{q}, J=7.1 \mathrm{~Hz}, 4 \mathrm{H}), 5.37(\mathrm{~s}, 1 \mathrm{H}), 6.14-6.21(\mathrm{~m}, 2 \mathrm{H}), 7.53$ $(\mathrm{m}, 1 \mathrm{H}), 7.70-8.16(\mathrm{~m}, 4 \mathrm{H}) ;{ }^{13} \mathrm{C}-\mathrm{NMR}\left(\mathrm{d}_{6}\right.$-DMSO) $\delta 15.22,19.01,33.75,61.87,104.63,107.34$, $111.02,118.93,123.40,137.66,143.40,146.58,147.52,149.42,153.55,167.09$. Anal. Calcd for $\mathrm{C}_{22} \mathrm{H}_{24} \mathrm{~N}_{2} \mathrm{O}_{5}$ : C, 66.65; H, 6.10, N, 7.07. Found: C, 66.59; H, 6.01; N, 7.03.

2,6-Dimethyl-1-(4-hydroxyphenyl)-4-(2-furyl)-1,4-dihydropyridine-3,5-diethylcarboxy late (Entry 22, Table 4). Brown solid; mp 68-69 ${ }^{\circ} \mathrm{C}$; IR (KBr) 3412, 3055, 2970, 1715, 1540, 1472, 1330, 1190, $\mathrm{cm}^{-1}$; ${ }^{1} \mathrm{H}-\mathrm{NMR}\left(\mathrm{CDCl}_{3}\right) \delta 1.21(\mathrm{t}, J=7.1 \mathrm{~Hz}, 6 \mathrm{H}), 1.93(\mathrm{~s}, 6 \mathrm{H}), 4.18(\mathrm{q}, J=7.0 \mathrm{~Hz}, 4 \mathrm{H}), 5.26(\mathrm{~s}, 1 \mathrm{H}), 6.11-6.20$ $(\mathrm{m}, 2 \mathrm{H}), 6.83(\mathrm{~d}, J=8.1 \mathrm{~Hz}, 2 \mathrm{H}), 6.88(\mathrm{~d}, J=8.0 \mathrm{~Hz}, 2 \mathrm{H}), 7.52(\mathrm{~m}, 1 \mathrm{H}), 9.82(\mathrm{~s}, 1 \mathrm{H}) ;{ }^{13} \mathrm{C}-\mathrm{NMR}$ $\left(\mathrm{CDCl}_{3}\right) \delta 15.11,19.52,33.29,61.77,104.53,107.22,112.40,118.41,122.00,132.86,141.38,147.26$, 
152.73, 154.25, 167.58. Anal. Calcd for $\mathrm{C}_{23} \mathrm{H}_{25} \mathrm{NO}_{6}$ : C, 67.14; H, 6.12, N, 3.40. Found: C, 66.99; $\mathrm{H}$, $6.06 ; \mathrm{N}, 3.37$.

2,6-Dimethyl-4-styryl-1,4-dihydropyridine-3,5-dimethylcarboxylate (Entry 23, Table 4). Yellow solid; mp 119-122 ${ }^{\circ} \mathrm{C}$; IR (KBr) 3332, 2949, 1701, 1652, 1560, 1492, 1121, $743 \mathrm{~cm}^{-1} ;{ }^{1} \mathrm{H}-\mathrm{NMR}\left(\mathrm{CDCl}_{3}\right) \delta$ $2.35(\mathrm{~s}, 6 \mathrm{H}), 3.71(\mathrm{~s}, 6 \mathrm{H}), 4.54(\mathrm{~d}, J=7.2 \mathrm{~Hz}, 1 \mathrm{H}), 5.61$ (br s, 1H), $6.18(\mathrm{~m}, 2 \mathrm{H}), 7.13-7.39(\mathrm{~m}, 5 \mathrm{H})$; ${ }^{13} \mathrm{C}-\mathrm{NMR}\left(\mathrm{CDCl}_{3}\right) \delta 14.90,19.31,36.17,51.13,102.18,126.34,126.98,128.07,128.28,131.77$, 137.68, 145.43, 167.92. Anal. Calcd for $\mathrm{C}_{19} \mathrm{H}_{21} \mathrm{NO}_{4}$ : C, 69.71; $\mathrm{H}, 6.47, \mathrm{~N}, 4.28$. Found: $\mathrm{C}, 69.63 ; \mathrm{H}$, $6.42 ; \mathrm{N}, 4.23$.

2,6-Dimethyl-4-cyclohexyl-1,4-dihydropyridine-3,5-diethylcarboxylate (Entry 24, Table 4). White solid; mp $214{ }^{\circ} \mathrm{C}$; IR (KBr) 2996, 1770, 1465, 1378, $1240 \mathrm{~cm}^{-1}$; ${ }^{1} \mathrm{H}-\mathrm{NMR}\left(\mathrm{CDCl}_{3}\right) \delta 1.24(\mathrm{t}, J=6.9 \mathrm{~Hz}$, $6 \mathrm{H}), 1.32-1.77(\mathrm{~m}, 11 \mathrm{H}), 2.24(\mathrm{~s}, 6 \mathrm{H}), 3.85(\mathrm{~d}, J=5.8 \mathrm{~Hz}, 1 \mathrm{H}), 4.21(\mathrm{q}, J=8.1 \mathrm{~Hz}, 4 \mathrm{H}), 5.81$ (br s, $1 \mathrm{H}) ;{ }^{13} \mathrm{C}-\mathrm{NMR}\left(\mathrm{CDCl}_{3}\right) \delta 15.08,19.23,26.21,26.64,32.69,38.36,45.85,50.97,102.02,144.78$, 168.74. Anal. Calcd for $\mathrm{C}_{19} \mathrm{H}_{29} \mathrm{NO}_{4}$ : C, 68.03; H, 8.71, N, 4.18. Found: C, 68.05; H, 8.66; N, 4.12.

2,6-Dimethyl-4-isopropyl-1,4-dihydropyridine-3,5-dimethylcarboxylate (Entry 25, Table 4). Yellow solid; mp 164-166 ${ }^{\circ} \mathrm{C}$; IR (KBr) 3334, 1705, 1652, 1570, 1462, 1375, 1220, $910 \mathrm{~cm}^{-1}$; ${ }^{1} \mathrm{H}-\mathrm{NMR}$ $\left(\mathrm{CDCl}_{3}\right) \delta 0.75(\mathrm{~d}, J=6.9 \mathrm{~Hz}, 6 \mathrm{H}), 1.64(\mathrm{sep}, J=6.8 \mathrm{~Hz}, 1 \mathrm{H}), 2.31(\mathrm{~s}, 6 \mathrm{H}), 3.72(\mathrm{~s}, 6 \mathrm{H}), 3.92(\mathrm{~d}$, $J=5.5 \mathrm{~Hz}, 1 \mathrm{H}), 5.65(\mathrm{~s}, 1 \mathrm{H}) ;{ }^{13} \mathrm{C}-\mathrm{NMR}\left(\mathrm{CDCl}_{3}\right) \delta 19.31,19.96,35.86,39.04,51.09,102.53,145.22$, 169.10. Anal. Calcd for $\mathrm{C}_{14} \mathrm{H}_{17} \mathrm{NO}_{4}$ : C, 62.90; H, 7.92, N, 5.24. Found: C, 62.78; H, 7.84; N, 5.17.

\section{Conclusions}

Application of microwave technology to rapid synthesis of biologically significant heterocyclic molecules under solvent-free conditions is very promising and challenging. The ultimate aim, of course, is to use no solvent at all and to conduct the reactions under solvent-free conditions [74]. Development of cleaner technologies is a major emphasis in green chemistry. The combination of solvent-free reaction conditions and microwave-irradiation is used as an eco-friendly approach for the synthesis of a variety of products and this generally leads to large reductions in reaction times, enhancements of conversions, and changes of selectivity.

There is growing interest in the one-pot three component synthesis of 1,4-dihydropyridines because of the significant importance of this scaffold in preparing a wide variety of biologically and pharmacologically active molecules. On this basis we have developed an extremely rapid, convenient and environmentally benign route for the one-step synthesis of 1,4-dihydropyridines. The present methodology offers attractive features such as shorter reaction times, milder conditions, and simplicity of the reaction as well as excellent yield of the products. This reaction will be applicable to the synthesis of various organic compounds of medicinal interest.

\section{Acknowledgments}

We gratefully acknowledge the funding support from Kleberg Foundation of Texas and NCI (NIH/NCI-P20, Grant\# 5P20CA138022-02). 


\section{References and Notes}

1. Dömling, A. Recent developments in isocyanide based multicomponent reactions in applied chemistry. Chem. Rev. 2006, 106, 17-89.

2. Ramón, D.J.; Yus, M. Asymmetric multicomponent reactions (AMCRs): The new frontier. Angew. Chem. Int. Ed. 2005, 44, 1602-1634.

3. Guillena, G.; Ramón, D.J.; Yus, M. Organocatalytic enantioselective multicomponent reactions (OEMCRs). Tetrahedron: Asymmetry 2007, 18, 693-700.

4. Isambert, N.; Duque, M.M.S.; Plaquevent, J.-C.; Genisson, Y.; Rodriguez, J.; Constantieux, T. Multicomponent reactions and ionic liquids: A perfect synergy for eco-compatible heterocyclic synthesis. Chem. Soc. Rev. 2007, 40, 1347-1357.

5. Duque, M.M.S.; Allais, C.; Isambert, N.; Constantieux, T.; Rodriguez, J. $\beta$-Diketo building blocks for MCRs-based syntheses of heterocycles. Top. Heterocycl. Chem. 2010, 23, 227-277.

6. Bonne, D.; Coquerel, Y.; Constantieux, T.; Rodriguez, J. 1,3-Dicarbonyl compounds in stereoselective domino and multicomponent reactions. Tetrahedron: Asymmetry 2010, 21, 1085-1109.

7. Hantzsch, A. Condensationprodukte aus Aldehydammoniak und ketoniartigen Verbindungen. Chem. Ber. 1881, 14, 1637-1638.

8. Merck Research Laboratories: The Merck Index, 12th ed.; Rahway, NJ, USA, 1996; p. 1121.

9. Boström, S.L.; Ljung, B.; Mårdh, S.; Forsen, S.; Thulin, E. Interaction of the antihypertensive drug felodipine with cal-modulin. Nature 1981, 292, 777-778.

10. Iwanami, M.; Shibanuma, T.; Fujimoto, M.; Kawai, R.; Tamazawa, K.; Takenaka, T.; Takahashi, K.; Murakami, M. Synthesis of new water-soluble dihydropyridine vasodilatators. Chem. Pharm. Bull. 1979, 27, 1426-1440.

11. Arrowsmith, J.E.; Campbell, S.F.; Cross, P.E.; Stubbs, J.K.; Burges, R.A.; Gardiner, D.G.; Blackburn, K.J. Long-acting dihydropyridine calcium antagonists. 1, 2-alkoxymethyl derivatives incorporating basic substituents. J. Med. Chem. 1986, 29, 1696-1702.

12. Harper, J.L.; Camerini-Otero, C.S.; Li, A.H.; Kim, S.A.; Jacobson, K.A.; Daly, J.W. Dihydropyridines as inhibitors of capacitative calcium entry in Leukemic HL-60 cells. Biochem. Pharmacol. 2003, 65, 329-338.

13. Zarghi, A.; Sadeghi, H.; Fassihi, A.; Faizi, M.; Shafiee, A. Synthesis and calcium antagonist activity of 1,4-dihydropyridines containing phenylaminoimidazolyl substituents. Farmaco 2003, 58, 1077-1081.

14. Peri, R.; Padmanabhan, S.; Singh, S.; Rutledge, A.; Triggle, D.J. Permanently charged chiral 1,4dihydropyridines: Molecular probes of L-type calcium channels. Synthesis and pharmacological characterization of methyl(-trimethylalkylammonium) 1,4-dihydro-2,6-dimethyl-4-(3nitrophenyl)-3,5- pyridinedicarboxylate iodide, calcium channel antagonists. J. Med. Chem. 2000, 43, 2906-2914.

15. Berkels, B.; Roesen, R.; Dhein, S.; Fricke, U.; Klaus, W. Dihydropyridine calcium antagonistinduced modulation of endothelial function: A review. Cardiovasc. Drug Rev. 1999, 17, 179-186.

16. Tsuruo, T.; Iida, H.; Nojiri, M.; Tsukagoshi, S.; Sakurai, Y. Circumvention of vincristine and adriamycin resistance in vitro and in vivo by calcium influx blockers. Cancer Res. 1983, 43, 2905-2910. 
17. Krauze, A.; Germane, S.; Eberlins, O.; Sturms, I.; Klusa, V.; Duburs, G. Derivatives of 3-cyano6-phenyl-4-(3'-pyridyl)pyridine-2(1H)-thione and their neurotropic activity. Eur. J. Med. Chem. 1999, 34, 301-310.

18. Zhou, X.; Zhang, L.; Tseng, E.; Scott-Ramsay, E.; Schentag, J.J.; Coburn, R.A.; Morris, M.E. New 4-aryl-1,4-dihydropyridines and 4-arylpyridines as P-glycoprotein inhibitors. Drug Metab. Dispos. 2005, 33, 321-328.

19. Chapman, R.W.; Danko, G.; Siegels, M.I. Effect of extra- and intracellular calcium blockers on histamine and antigen-induced bronchospasm in guinea pigs and rats. Pharmacology 1984, 29, 282-291.

20. Malaise, W.J.; Mathias, P.C.F. Stimulation of insulin release by organic calcium-agonist. Diabetologia 1985, 28, 153-156.

21. Long, S.; Panunzio, M.; Petroli, A.; Qin, W.; Xia, Z. The use of magnesium nitride for the synthesis of enantiomerically pure 1,4-dihydropyridines via the Hantzsch reaction. Synthesis 2011, 7, 1071-1078.

22. Vijesh, A.M.; Isloor, A.M.; Peethambar, S.K.; Shivananda, K.N.; Arulmoli, T.; Isloor, N.A. Hantzsch reaction. Synthesis and characterization of some new 1,4-dihydropyridine derivatives as potent antimicrobial and antioxidant agents. Eur. J. Med. Chem. 2011, 46, 5591-5597.

23. Saini, A.; Kumar, S.; Sandhu, J.S. Hantzsch reaction: Recent advances in Hantzsch 1,4-dihydropyridines. J. Sci. Ind. Res. 2008, 67, 95-111.

24. Bhatti, R.S.; Krishan, P.; Suresh; Sandhu, J.S. Bismuth trichloride, a clean, green catalyst for the synthesis of Hantzsch 1,4- dihydropyridines (DHPs). J. Indian Chem. Soc. 2010, 87, 707-710.

25. Kolvari, E.; Zolfigol, M.A.; Koukabi, N.; Shirmardi-Shaghasemi, B. A simple and efficient onepot synthesis of Hantzsch 1,4-dihydropyridines using silica sulphuric acid as a heterogeneous and reusable catalyst under solvent-free conditions. Chem. Papers 2011, 65, 898-902.

26. Koukabi, N.; Kolvari, E.; Khazaei, A.; Zolfigol, M.A.; Shirmardi-Shaghasemi, B.; Khavasi, H.R. Hantzsch reaction on free nano- $\mathrm{Fe}_{2} \mathrm{O}_{3}$ catalyst: Excellent reactivity combined with facile catalyst recovery and recyclability. Chem. Commun. 2011, 47, 9230-9232.

27. Ladani, N.K.; Mungra, D.C.; Patel, M.P.; Patel, R.G. Microwave-assisted synthesis of novel Hantzsch 1,4-dihydropyridines, acridine-1,8-diones and polyhydroquinolines bearing the tetrazolo[1,5-a]quinoline moiety and their antimicrobial activity assess. Chin. Chem. Lett. 2011, $22,1407-1410$.

28. Akbari, J.D.; Tala, S.D.; Dhaduk, M.F.; Joshi, H.S. Molecular iodine-catalyzed one-pot synthesis of some new Hantzsch 1,4-dihydropyridines at ambient temperature. ARKIVOC 2008, 12, 126-135.

29. Sureshkumar, D.; Sandhu, J.S. New efficient protocol for the production of Hantzsch 1,4-dihydropyridines using $\mathrm{RuCl}_{3}$. Synth. Commun. 2009, 39, 1957-1965.

30. Yadav, D.K.; Patel, R.; Srivastava, V.P.; Watal, G.; Yadav, L.D.S. LiBr as an efficient catalyst for one-pot synthesis of Hantzsch 1,4-dihydropyridines under mild conditions. Chin. J. Chem. 2011, 29, 118-122.

31. Kumar, B.R.P.; Masih, P.; Lukose, C.R.; Abraham, N.; Priya, D.; Xavier, R.M.; Saji, K.; Adhikary, L. Facile, microwave-assisted parallel syntheses of N-substituted 1,4-dihydropyridines of biological interest. J. Heterocycl. Chem. 2009, 46, 336-339. 
32. Ghorbani-Choghamarani, A.; Zolfigol, M.A.; Hajjami, M.; Goudarziafshar, H.; Nikoorazm, M.; Yousefi, S.; Tahmasbi, B. Nano aluminium nitride as a solid source of ammonia for the preparation of Hantzsch 1,4-dihydropyridines and bis-(1,4-dihydropyridines) in water via one pot multicomponent reaction. J. Braz. Chem. Soc. 2011, 22, 525-531.

33. Lee, J.H. Synthesis of Hantsch 1,4-dihydropyridines by fermenting bakers' yeast. Tetrahedron Lett. 2005, 46, 7329-7330.

34. Sathicq, A.G.; Romanelli, G.P.; Ponzinibbio, A.; Baronetti, G.T.; Thomas, H.J. An efficient onestep Hantzsch multicomponent synthesis of 1,4-dihydropyridines via a Wells-Dawson heteropolyacid catalyst under solvent-free conditions. Lett. Org. Chem. 2010, 7, 511-518.

35. Chari, M.A.; Syamasundar, K. Silica gel/NaHSO 4 catalyzed one-pot synthesis of Hantzsch 1,4dihydropyridines at ambient temperature. Catal. Commun. 2005, 6, 624-626.

36. Heydari, A.; Khaksar, S.; Tajbakhsh, M.; Bijanzadeh, H.R. One-step, synthesis of Hantzsch esters and polyhydroquinoline derivatives in fluoro alcohols. J. Fluorine Chem. 2009, 130, 609-614.

37. Legeay, J.-C.; Eyndeb, J.J.V.; Bazureaua, J.P. Ionic liquid phase technology supported the three component synthesis of Hantzsch 1,4-dihydropyridines and Biginelli 3,4-dihydropyrimidin2(1H)-ones under microwave dielectric heating. Tetrahedron 2005, 61, 12386-12397.

38. Bandyopadhyay, D.; Banik, B.K. Microwave-induced stereoselectivity of $\beta$-lactam formation with dihydrophenanthrenyl imines via Staudinger cycloaddition. Helv. Chim. Acta 2010, 93, 298-301.

39. Bandyopadhyay, D.; Yañez, M.; Banik, B.K. Microwave-induced stereoselectivity of $\beta$-lactam formation: effects of solvents. Heterocycl. Lett. 2011, 1, 65-67.

40. Bandyopadhyay, D.; Rivera, G.; Salinas, I.; Aguilar, H.; Banik, B.K. Iodine-catalyzed remarkable synthesis of novel $N$-polyaromatic $\beta$-lactams bearing pyrroles. Molecules 2010, 15, 1082-1088.

41. Bandyopadhyay, D.; Mukherjee, S.; Banik, B.K. An expeditious synthesis of $N$-substituted pyrroles via microwave-induced iodine-catalyzed reaction under solventless conditions. Molecules 2010, 15, 2520-2525.

42. Andoh-Baidoo, R.; Danso, R.; Mukherjee, S.; Bandyopadhyay, D.; Banik, B.K. Microwaveinduced $N$-bromosuccinimide-mediated novel synthesis of pyrroles via Paal-Knorr reaction. Heterocycl. Lett. 2011, 1, 107-109.

43. Bandyopadhyay, D.; Banik, A.; Bhatta, S.; Banik, B.K. Microwave-assisted ruthenium trichloride catalyzed synthesis of pyrroles fused with indole systems. Heterocycl. Commun. 2009, 15, 121-122.

44. Abrego, D.; Bandyopadhyay, D.; Banik, B.K. Microwave-induced indium-catalyzed synthesis of pyrrole fused with indolinone in water. Heterocycl. Lett. 2011, 1, 94-95.

45. Kall, A.; Bandyopadhyay, D.; Banik, B.K. Microwave-induced aza-Michael reaction in water: A remarkable simple procedure. Synth. Commun. 2010, 42, 1730-1735.

46. Bandyopadhyay, D.; Mukherjee, S.; Rodriguez, R.R.; Banik, B.K. An effective microwaveinduced iodine-catalyzed method for the synthesis of quinoxalines via condensation of 1,2-dicarbonyl compounds. Molecules 2010, 15, 4207-4212.

47. Bothwell, J.M.; Krabbe, S.W.; Mohan, R.S. Applications of bismuth(iii) compounds in organic synthesis. Chem. Soc. Rev. 2011, 40, 4649-4707.

48. Hua, R. Recent advances in bismuth-catalyzed organic synthesis. Curr. Org. Synth. 2008, 1, 1-27. 
49. Salvador, J.A.R.; Pinto, R.M.A.; Silvestre, S.M. Recent advances of bismuth(III) salts in organic chemistry: Application to the synthesis of aliphatics, alicyclics, aromatics, amino acids and peptides, terpenes and steroids of pharmaceutical interest. Mini Rev. Org. Chem. 2009, 6, 241-274.

50. Salvador, J.A.R.; Pinto, R.M.A.; Silvestre, S.M. Recent advances of bismuth(III) salts in organic chemistry: Application to the synthesis of heterocycles of pharmaceutical interest. Curr. Org. Synth. 2009, 6, 426-470.

51. Canales, L.; Bandyopadhyay, D.; Banik, B.K. Bismuth nitrate pentahydrate-induced novel nitration of Eugenol. Org. Med. Chem. Lett. 2011, 1, 9.

52. Banik, B.K.; Samajdar, S.; Banik, I.; Ng, S.; Hann, J. Montmorillonite impregnated with bismuth nitrate: Microwave-assisted facile nitration of $\beta$-lactams. Heterocycles 2003, 61, 97-100.

53. Bose, A.; Sanjoto, W.P.; Villarreal, S.; Aguilar, H.; Banik, B.K. Novel nitration of estrone by metal nitrates. Tetrahedron Lett. 2007, 48, 3945-3947.

54. Srivastava, N.; Banik, B.K. Bismuth nitrate-catalyzed versatile Michael reactions. J. Org. Chem. 2003, 68, 2109-2114.

55. Srivastava, N.; Dasgupta, S.K.; Banik, B.K. A remarkable bismuth nitrate-catalyzed protection of carbonyl compounds. Tetrahedron Lett. 2003, 44, 1191-1193.

56. Banik, B.K.; Adler, D.; Nguyen, P.; Srivastava, N. A new bismuth nitrate-induced stereospecific glycosylation of alcohols. Heterocycles 2003, 61, 101-104.

57. Rivera, S.; Bandyopadhyay, D.; Banik, B.K. Facile synthesis of $N$-substituted pyrroles via microwave-induced bismuth nitrate-catalyzed reaction under solventless conditions. Tetrahedron Lett. 2009, 50, 5445-5448.

58. Bandyopadhyay, D.; Fonseca, R.S.; Banik, B.K. Microwave-induced bismuth nitrate-mediated selective hydrolysis of amide. Heterocycl. Lett. 2011, 1, 75-77.

59. Iglesias, L.; Aguilar, C.; Bandyopadhyay, D.; Banik, B.K. A new bismuth nitrate-catalyzed electrophilic substitution of indoles with carbonyl compounds under solventless conditions. Synth. Commun. 2010, 40, 3678-3682.

60. Rivera, S.; Bandyopadhyay, D.; Banik, B.K. Microwave-induced bismuth nitrate-catalyzed electrophilic substitution of 7-aza indole with activated carbonyl compound under solvent-free conditions. Heterocycl. Lett. 2011, 1, 43-46.

61. Banik, A.; Bhatta, S.; Bandyopadhyay, D.; Banik, B.K. A highly efficient bismuth salts-catalyzed route for the synthesis of $\alpha$-aminophosphonates. Molecules 2010, 15, 8205-8213.

62. Banik, B.K.; Reddy, A.T.; Datta, A.; Mukhopadhyay, C. Microwave-induced bismuth nitratecatalyzed synthesis of dihydropyrimidones via Biginelli condensation under solventless conditions. Tetrahedron Lett. 2007, 48, 7392-7394.

63. Tajbakhsh, M.; Heravi, M.M.; Hosseini, A.; Shahrezaiee, A. Old reagent, new results: Aromatization of Hantzsch 1,4-dihydropyridines with supported bismuth nitrate under microwave irradiation in solventless system. Phosphor. Sulfur Silicon 2003, 178, 773-776.

64. Becker, F.F.; Banik, B.K. Polycyclic aromatic compounds as anticancer agents: Synthesis and biological evaluation of some chrysene derivatives. Bioorg. Med. Chem. Lett. 1998, 8, 2877-2880.

65. Becker, F.F.; Mukhopadhyay, C.; Hackfeld, L.; Banik, I.; Banik, B.K. Polycyclic aromatic compounds as anticancer agents: synthesis and biological evaluation of dibenzofluorene derivatives. Bioorg. Med. Chem. 2000, 8, 2693-2699. 
66. Banik, B.K.; Becker, F.F. Polycyclic aromatic compounds as anticancer agents. 4. Structureactivity relationships of chrysene and pyrene derivatives. Bioorg. Med. Chem. 2001, 9, 593-605.

67. Banik, B.K.; Becker, F.F. Synthesis, electrophilic substitution and structure-activity relationship studies of polycyclic aromatic compounds towards the development of anticancer agents. Curr. Med. Chem. 2001, 8, 1513-1533.

68. Banik, B.K.; Becker, F.F.; Banik, I. Synthesis of anticancer $\beta$-lactams: Mechanism of action. Bioorg. Med. Chem. 2004, 12, 2523-2528.

69. Banik, I.; Becker, F.F.; Banik, B.K. Stereoselective synthesis of $\beta$-lactams with polyaromatic imines: Entry to new and novel anticancer agents. J. Med. Chem. 2003, 46, 12-15.

70. Bandyopadhyay, D.; Granados, J.C.; Short, J.D.; Banik, B.K. Polycyclic aromatic compounds as anticancer agents: evaluation of synthesis and in vitro cytotoxicity. Oncol. Lett. 2012, 3, 45-49.

71. Blackwell, H.E. Out of the oil bath and into the oven-Microwave-assisted combinatorial chemistry heats up. Org. Biomol. Chem. 2003, 1, 1251-1255.

72. Kidwai, M. Green chemistry trends toward sustainability. Pure Appl. Chem. 2006, 78, 1983-1992.

73. Kranjc, K.; Kočevar, M. Microwave-assisted organic synthesis: General considerations and transformations of heterocyclic compounds. Curr. Org. Chem. 2010, 14, 1050-1074.

74. Martins, M.A.P.; Frizzo, C.P.; Moreira, D.N.; Buriol, L.; Machado, P. Solvent-free heterocyclic synthesis. Chem. Rev. 2009, 109, 4140-4182.

Sample Availability: Samples of the all compounds (mg quantity) are available from the authors.

(C) 2012 by the authors; licensee MDPI, Basel, Switzerland. This article is an open access article distributed under the terms and conditions of the Creative Commons Attribution license (http://creativecommons.org/licenses/by/3.0/). 